\title{
Requiem for Robotic Cancer Surgery? Not So Fast
}

\author{
John D. Seigne, $\mathrm{MD}^{1}$, Ilana Cass, $\mathrm{MD}^{2}$, and Sandra L. Wong, MD, $\mathrm{MS}^{3}$ \\ ${ }^{1}$ Section of Urology, Department of Surgery, Dartmouth-Hitchcock Medical Center, Lebanon, NH; ${ }^{2}$ Department of \\ Obstetrics-Gynecology, Dartmouth-Hitchcock Medical Center, Lebanon, NH; ${ }^{3}$ Department of Surgery, Dartmouth- \\ Hitchcock Medical Center, Lebanon, NH
}

Recently, the US FDA cautioned against the use of robotic surgery in cancer operations, stating that "the use of robotically-assisted surgical devices for any cancer-related surgery has not been granted marketing authorization," and that "survival benefits to patients when compared to traditional surgery have not been established". ${ }^{1}$ Several watchdog groups called for a moratorium on robotic cancer surgery. Although the FDA warning was in part prompted by two studies that showed inferior oncologic outcomes for minimally invasive surgery (MIS) for early cervical cancer, the expansive nature of the FDA communication included comment on new (and uncommon) innovations, such as robot-assisted mastectomy and robotic cancer surgery writ large.

\section{LESSONS FROM CERVICAL CANCER}

Robotic surgery gained acceptance across many specialists due to its superior visualization, improved dexterity, and surgical precision. A survey of members of the Society of Gynecologic Oncologists found that $97 \%$ of respondents reported using the robot in 2012, compared with only $29 \%$ in 2007 . There was a significant increase in the perceived appropriateness of robotic surgery for radical hysterectomy and lymph node dissection for the management of cervical cancer over time, from $60.2 \%$ in 2007 to $89.1 \%$ in 2012 . The robot was favored over traditional laparoscopy for cervical cancer in $75 \%$ of respondents by

(C) Society of Surgical Oncology 2019

First Received: 17 July 2019;

Published Online: 2 August 2019

S. L. Wong, MD, MS

e-mail: Sandra.L.Wong@hitchcock.org
2012. ${ }^{2}$ As surgeons continued to adopt the technology, prospective studies were designed to definitively answer the question regarding the optimal surgical approach for cervical cancer. Two companion articles published in the New England Journal of Medicine raised immediate concerns about the safety of the minimally invasive approach compared with open surgery for the management of early cervical cancer. The Laparoscopic Approach to Cervical Cancer (LACC) trial was an international, multicenter, randomized, phase III trial of women with stage IA1 (with lymphovascular space invasion), IA2, or IB1 squamous, adenosquamous, or adenocarcinoma of the uterine cervix, to test for non-inferiority of minimally invasive radical hysterectomy compared with open radical hysterectomy. ${ }^{3}$

The LACC trial was stopped early due to the finding that women randomized to minimally invasive radical hysterectomy had four times as many cancer recurrences and six times as many deaths as women randomized to open radical hysterectomy. Noting a preponderance of laparoscopic cases, compared with robotic cases, a per-protocol analysis was performed and supported the same findings for the women treated with robotic surgery as those treated with conventional laparoscopic surgery. A companion retrospective study using the Surveillance, Epidemiology, and End Results (SEER) database mirrored the adverse outcome for women treated with MIS compared with open surgery, with $80 \%$ of women in the minimally invasive arm treated with robotic radical hysterectomy. ${ }^{4}$ In a subgroup analysis, robotic and laparoscopic surgery were both associated with a higher risk of death than open surgery. Updated findings from the LACC trial confirm that the inferior outcomes observed in the MIS arm cannot be attributed to lower-volume centers or surgeons. Similarly, concerns that selection bias may have influenced the superior oncologic outcome of women in the open arm has been addressed by additional updated data that showed 
more women in the open arm had poorer prognosis tumors, although they had comparable rates of adjuvant treatment following surgery. ${ }^{5}$

The underlying mechanism that drives the poorer outcome of laparoscopic and robotic surgery in cervical cancer has yet to be identified, although the collective findings have largely halted minimally invasive radical hysterectomy for cervical cancer. With due credit to the gynecologic oncologists who organized a randomized controlled trial (RCT) to study open versus minimally invasive radical hysterectomy, the development of robotassisted surgery for other types of cancer has not followed a similarly strict evidence-based approach.

\section{Buxton's Law: "It is always too early (for rigorous evaluation) until it is suddenly too late". 6}

The optimal development and introduction of a new surgical tool or procedure should include randomized trials or follow a stepwise process such as that recommended in the IDEAL (Idea, Development, Exploration, Assessment, Long-term study) framework, which is a systematic method for the development, introduction and evaluation of new surgical procedures, invasive medical devices, and complex therapeutic interventions. ${ }^{7}$ IDEAL emphasizes appropriate methods, transparency of data, and rigorous reporting of outcomes. However, direct application of this framework to procedures or instruments already in use is challenging as practice often moves faster than data.

Understanding the exact nature of the change and to what degree the change has the potential to affect outcomes, is paramount when deciding how best to evaluate a change in surgical practice. There are likely more questions than answers at this juncture of ongoing scrutiny by federal agencies and the public. Do we need an RCT to show the benefit of a new type of right angle to divide the dorsal vein complex at the time of open radical prostatectomy or a new lighted retractor to improve visualization while performing an abdominoperineal resection? Is it okay to allow a surgeon to use different instruments that make certain portions of an operation easier or more effective to perform? Is the robot just another instrument like a new right angle or new retractor (albeit substantially more expensive and complex), and how are factors such as surgeon experience and judgment weighed when assessing technology? When and to what degree do we need to scientifically assess the tool (here, the robot) when we have data that the approach (minimally invasive laparoscopic surgery) provides equivalent oncological results?

The publication of the LACC trial raises legitimate questions about the oncological efficacy of minimally invasive hysterectomy for the treatment of cervical cancer (2); however, we need to be careful about generalizing these results to other minimally invasive cancer operations. There is good evidence for oncological noninferiority of MIS and robotic surgery in other tumor types. For instance, in colorectal surgery, several randomized trials $\left(\mathrm{COST},{ }^{8}\right.$ CLASICC, ${ }^{9}$ and $\mathrm{COLOR}^{10}$ ) have shown an improved postoperative recovery and similar rates of cancer recurrence, complications, and long-term survival between the open and laparoscopic groups. In the area of rectal cancer, where there is more controversy about the oncological outcomes of MIS, two small randomized trials have shown improvement in the length of tumor-free distal resection margins with the use of a surgical robot. ${ }^{11,12}$ In the case of bladder cancer, the RAZOR trial ${ }^{13}$ showed that robot-assisted cystectomy was noninferior to an open cystectomy with regard to 2-year progression-free survival.

Complicating the assessment of the value of MIS, and specifically robotics in cancer surgery, is that much of the early development was performed in procedures in which the recovery and complication rates are in general quite low, so that although one can debate oncological efficacy, perhaps the focus should have been more on the low level of benefit that the added cost of robotic surgery provides. For example, the first randomized trial comparing open and robotic prostatectomy was published in 2016 and demonstrated similar functional outcomes between the two techniques. ${ }^{14}$ Unfortunately, it took more than 15 years from the introduction of robotic prostatectomy to publication of these results, and, by that time, $>85 \%$ of prostatectomies in the US were being performed robotically.

Although as yet untested in RCTs for all sites, the value of robotic cancer surgery is more clearly seen in procedures where a minimally invasive approach avoids large morbid incisions, and rapid, precise dissection and suturing are needed for a successful operation, for example in the procedures of partial nephrectomy and lung resections. In such procedures where the decrease in morbidity is clear, unusual patterns of recurrence are rarely observed, and cohort studies show similar outcomes, practical and science-informed considerations of patient benefit and oncological equivalence must be balanced with a need for randomized trials.

\section{EVALUATION OF INNOVATION}

The lessons of the LACC trial point out the need for a more structured approach to the expansion of robotic cancer surgery. The FDA communication appropriately draws attention to the need for careful examination of a new technique (robotic mastectomy) in the management of a cancer for which open surgery has been so carefully studied. The Dutch experience with robotic esophagectomy 
(RAMIE) demonstrates how the IDEAL framework can be used to appropriately develop, test, introduce, and disseminate a new cancer surgery. ${ }^{15}$ As current procedures are converted from open to laparoscopic to robotic, and new robots become available, it will be important to determine how different these new techniques are from our current standard. Is a formal IDEAL process required? When would a registry approach suffice to detect unexpected adverse effects? Surgeons taking responsibility for developing groups within institutions and national surgical societies to create consensus and provide guidelines will help ensure safe, structured, and data-driven development and introduction of new procedures. We must continue to innovate, but there should be no innovation without evaluation.

DISCLOSURE John D. Seigne has common stock holdings in Johnson \& Johnson.

\section{REFERENCES}

1. US Food and Drug Administration. Date issued 28 February 2019. https://www.fda.gov/medical-devices/safety-communicatio ns/caution-when-using-robotically-assisted-surgical-devices-wo mens-health-including-mastectomy-and

2. Conrad LB, Ramirez PT, Burke W, et al. Role of minimally invasive surgery in gynecologic oncology: an updated survey of members of the Society of Gynecologic Oncology. Int J Gynecol Cancer. 2015:25(6):1121-7.

3. Ramirez PT, Frumowitz M, Pareja R, et al Minimally invasive versus abdominal radical hysterectomy for cervical cancer. $N$ Engl J Med. 2018;379(20):1895-904.

4. Melamed A, Margul DJ, Chen LC, et al Survival after minimally invasive radical hysterectomy for early-stage cervical cancer. $N$ Engl J Med. 2018:379(20):1905-14.

5. Ramirez PT. LACC Trial Update. 50th Annual Meeting on Women's Cancer, Honolulu, Hawaii; March 2019.
6. Groves T. IDEAL innovation in surgery. The BMJ Opinion. 17 December 2010. https://blogs.bmj.com/bmj/2010/12/17/trish-gro ves-ideal-innovation-in-surgery-2/.

7. The IDEAL Collaboration. http://www.ideal-collaboration.net/

8. Fleshman J, Sargent DJ, Green E, Anvari M, Stryker SJ, Beart $\mathrm{RW} \mathrm{Jr}$, et al. Laparoscopic colectomy for cancer is not inferior to open surgery based on 5-year data from the COST Study Group trial. Ann Surg. 2007;246(4):655-62.

9. Guillou PJ, Quirke P, Thorpe H, Walker J, Jayne DG, Smith AM, et al. Short-term endpoints of conventional versus laparoscopicassisted surgery in patients with colorectal cancer (MRC CLASICC trial): multicentre, randomised controlled trial. Lancet. 2005;365(9472):1718-26.

10. COLOR Study Group. COLOR: a randomized clinical trial comparing laparoscopic and open resection for colon cancer. Dig Surg. 2000;17(6):617-22.

11. Somashekhar SP, Ashwin KR, Rajashekhar J, Zaveri S. Prospective randomized study comparing robotic-assisted surgery with traditional laparotomy for rectal cancer-indian study. Ind $J$ Surg. 2015;77 Suppl 3:788-94.

12. Baik SH, Kwon HY, Kim JS, Hur H, Sohn SK, Cho CH, et al. Robotic versus laparoscopic low anterior resection of rectal cancer: short-term outcome of a prospective comparative study. Ann Surg Oncol. 2009;16(6):1480-7.

13. Parekh DJ, Reis IM, Castle EP, Gonzalgo ML, Woods ME, Svatek RS, et al. Robot-assisted radical cystectomy versus open radical cystectomy in patients with bladder cancer (RAZOR): an open-label, randomised, phase 3, non-inferiority trial. Lancet. 2018;391(10139):2525-36.

14. Yaxley JW, Coughlin GD, Chambers SK, Occhipinti S, Samaratunga $\mathrm{H}$, Zajdlewicz L, et al. Robot-assisted laparoscopic prostatectomy versus open radical retropubic prostatectomy: early outcomes from a randomised controlled phase 3 study. Lancet. 2016;388(10049):1057-66.

15. van der Sluis PC, van Hillegersberg R. Robot assisted minimally invasive esophagectomy (RAMIE) for esophageal cancer. Best Pract Res Clin Gastroenterol. 2018;36-37:81-3.

Publisher's Note Springer Nature remains neutral with regard to jurisdictional claims in published maps and institutional affiliations. 